\title{
Categorizaciones de la lectura y praxis cultural en la era digital: distant reading vs. close reading
}

\author{
Eloy Martos Núñez \\ Aitana Martos García*
}

Artículo recibido:

28 de mayo de 2015

Artículo aceptado:

15 de abril de 2016

\section{Resumen}

El artículo examina los conceptos de distant reading y close reading en función del contexto de abundancia de información y de los distintos fenómenos de la praxis cultural de la era digital, desde los modelos educativos clásicos hasta las modernas corrientes culturales. Revisa las aproximaciones de Franco Moretti y otros autores. Concluye que en el actual contexto de hibridación y de globalizacion de contenidos, la escuela y la biblioteca deben aprender de las prácticas culturales alternativas y hacer un esfuerzo por recuperar la lectura intensiva bajo otros supuestos y con otras estrategias.

* Universidad de Extremadura, España

emartos@unex.es

** Universidad de Almería, España

aitmartos@gmail.com 
Palabras clave: Distant Reading; Close Reading;

Texto; Biblioteca; Escuela

Categorizations of reading and cultural praxis in the digital age: distant reading vs. close reading Eloy Martos-Núñez, Aitana Martos-García

\begin{abstract}
Through a review of the work of Franco Moretti and others, this paper examines the concept of distant reading and close reading in recognition of abundant information and diverse phenomena of cultural praxis of the digital age, ranging from the traditional education models to modern cultural currents. The paper concludes that in the current context of globalization and hybridization of contents, the school and the library should learn from alternative cultural practices and strive to return to intensive reading under other assumptions and other strategies.
\end{abstract}

Keywords: Distant Reading; Close Reading; Text; Library; School

\title{
INTRODUCCIÓN: LOS NUEVOS CONTEXTOS TEÓRICOS Y LAS NUEVAS CORRIENTES CULTURALES
}

T a sociedad de la información ha producido un hiato claro en las prácticas Lde lectura pero también en sus paradigmas teóricos. Por ejemplo, en la tradición escolar francesa del siglo XIX y gran parte del XX, instrumentos como la explicación de textos, la lectura intensiva o el comentario de textos eran métodos recurrentes de aproximación teórica-práctica. De tal modo, leer se equiparaba a hacer close reading, denominada entonces lectura comentada o análisis textual, reducida a que el profesor explicaba el sentido de ciertos fragmentos elegidos de los textos canónicos, proporcionando a los alumnos todas las claves y el "sentido correcto" de los mismos. Este aparato crítico contenía información literaria, histórica, retórica, etc., y lo que se pedía después a los alumnos era que retuviesen estas informaciones y, a lo sumo, que 
emulasen estos procedimientos ya prestablecidos del texto (Dufays, Gemenne y Ledur, 2005: 25).

El principal problema que pone en cuestión todo este modelo es la abundancia o saturación de información, que distorsiona muchas de estas situaciones. Así, un profesor de literatura infantil y juvenil se enfrenta a una multiplicación exponencial de lecturas disponibles (por ejemplo, títulos publicados de un género concreto, en forma de clásicos, fantasía, ciencia ficción o género histórico). Por otra parte, el propio canon clásico de lecturas está en crisis, porque ya no está claro de si hablamos de una literatura a nivel local, nacional o mundial, unilingüe o multilingüe, "occidental" o intercultural. De hecho, estamos ante un paradigma nuevo que Jenkins (2006) ha descrito acertadamente como cultura de la convergencia y la participación.

El mundo de los textos clásicos ya no se parece, entonces, a un mar en calma con una serie de referentes incuestionables, sino más bien a una continua marejada provocada sobre todo por las corrientes que circulan en el "océano de la información", que es Internet y la cibercultura. Así, la educación lectora ya no se puede separar de la cultura mediática y digital, ni de la propia infraestructura de la industria del entretenimiento. Las historias ya no se cuentan simplemente por vía oral o a través de los libros impresos, sino a través de numerosos lenguajes y soportes, que constituyen lo que se ha dado en llamar el universo transmedia.

Por todo ello, se hace necesario el procesamiento masivo de datos, y por eso Franco Moretti (2013) ha postulado el concepto de distant reading como una forma de distanciarnos del objeto en sí y de tratar de tener una visión de conjunto que permita hallar patrones y constantes significativas. Esta posición supone invertir el foco respecto a la escuela tradicional, porque en el fondo ya no estamos simplemente ante expresiones de cine, libros o televisión sino, cada vez más, ante bases de datos (Lovink, 2013). Es el caso de TVTropes, que nos organiza un auténtico "ficcionario" (tvtropes.org) ${ }^{1}$ (Martos Núñez y Martos García, 2014). Esta abundancia de datos ha llevado a demandas cada vez más intensas de descentralización, de colaboración y de publicación en abierto.

Por otro lado, hablamos de Internet como "océano de la información" más allá de una mera metáfora: la idea del texto líquido y de la modernidad líquida (Martos Núñez y Martos García, 2014) supone reconceptualizar el texto no como un discontinuo, una obra acotada, un volumen o

1 TVTropes.org es una wiki angloparlante basada en la escritura de ficción. Contiene páginas para numerosas películas, libros y otros elementos, y los asocia con tropos (dispositivos de escritura y convenciones). DBTropes.org es una Linked Data para TVTropes.org. Para formalizar items y tropos, es usada la ontología de Skipinions, creada en Skipforward. 
pieza bibliográfica, sino como un continuum creativo que en efecto se puede descomponer y recomponer, no ya en los capítulos clásicos de un libro sino en episodios, en antecedentes y prolongaciones (precuelas y secuelas), en una variedad de fragmentos que no ya el autor sino el editor o incluso el lector es capaz de "integrar" dándole un sentido nuevo.

En efecto, la problemática de los textos integrados es actualmente muy significativa (Martos Núñez y Martos García, 2014), porque este mundo textual en continua expansión es por definición inestable -a diferencia del corpus textual impreso- y sólo puede ser percibido si se le "trocea" en partes -como las pantallas de un hipertexto-, que luego puedan ser reordenadas de diferentes modos y con diferentes propósitos. Así, una antología de historias de terror supone hilvanar toda una serie de tradiciones, autores y textos, papel que antes hacía casi con exclusividad un compilador o un editor, pero que ahora llevan a cabo más agentes, por ejemplo, una comunidad de fans (fandom).

En el fondo de estas transformaciones lo que hay es una praxis cultural radicalmente distinta: la lectura ya no es una simple práctica de formación humanística sino una práctica de consumo en toda su acepción; se acomoda, pues, al mercado, y participa del mismo marketing, modas y presiones propias de la industria de la lectura con sus nuevos soportes y tecnologías. El narrador clásico, por ejemplo, se ha transformado en un narrador transmedia o en un storyteller digital cuya historia es "remediada" continuamente (Bolter, Grusin y Grusin, 2000), es decir, salta de un formato a otro, aprovechando lo que Besson (2004) llama la porosidad de estos universos de ficción.

De este modo, lo que estaba en el libro de texto impreso clásico ahora "salta" a otras plataformas y lenguajes, y, por tanto, la educación lectora y literaria clásica se va transformando cada vez más en una suerte de educación del consumidor, de promover no ya el lector culto propio de Chartier (1992), de la cultura letrada, sino al menos un lector informado, capaz de (ob)tener criterios por sí mismo para elegir y defenderse de estereotipos o de ciertas lecturas "tóxicas". Por ello mismo, la lectura literaria no sólo debe convertirse en un ingrediente de la educación del consumidor sino de la propia alfabetización ciudadana (Morin y Pakman, 1994), como un medio de aprehender esa cultura compleja y de escapar de los reduccionismos y dicotomías entre, por ejemplo, las ciencias experimentales y las humanidades. 


\section{Las Humanidades Digitales y Moretti}

Las llamadas Humanidades Digitales han tratado de usar el texto como campo de esta aproximación. Entre sus investigadores más destacados hay que citar al profesor Moretti (2005), cuyas teorías han servido cuando menos de revulsivo. Por ejemplo, las investigaciones cuantitativas son esclarecedoras para ponderar si un género literario está en decadencia o en expansión, y arroja siempre resultados que contextualizan la obra literaria. Lo que afirma Moretti (2013) es que para entender los textos en cierto modo hay que dejar de leer, esto es, de centrarse en la "unicidad" que pueda suponer una lectura, por ejemplo, el Quijote, para situarnos en el flujo de la corriente de textos en que una obra está inserta.

Como acabamos de enunciar, Franco Moretti es un experto en literatura comparada que promueve una aproximación singular a los textos, como ha sido la cartografía de la novela usando los mapas como instrumentos analíticos (Moretti, 2005). En el caso de la literatura vista "desde lejos", el enfoque es cuantitativo, en oposición a la close reading del New Criticism que se apoyaba en el canon.

La paradoja está en que la close reading se acerca mucho a lo que conocemos como lectura erudita o lectura intensiva, y que la distant reading podría equiparase en principio a un modo extensivo de lectura, a una lectura comunitaria que no se fija sino en ciertas informaciones "superficiales", por ejemplo, las sinopsis con que todas las páginas de Internet tratan de hacer una especie de booktrailer. Sin embargo, la intención de Moretti (2000) es generar un distanciamiento que es equidistante a los métodos tradicionales de la inducción o la deducción, se parece más bien a la transducción, en el sentido de pretender que el material analizado con estos métodos nos ayude a construir nuevas categorías y aproximaciones.

Puede parecer que esta lectura alejada privilegia la reducción y la abstracción en detrimento de la interpretación, pero eso ya venía ocurriendo con otros métodos más clásicos, por ejemplo, las concordancias de santa Teresa (o de otros autores clásicos) suponía un trabajo intenso de inventariar todo un aparato de citas y expresiones de la autora para revelar perfiles insospechados a veces, nociones que antes habían pasado inadvertidas a la crítica o que podían estar testimoniadas en escritos menores, como cartas u otros documentos. Extraídos estos datos, lo que hace Moretti (2005) es visualizarlos en 
formatos analíticos, como mapas, tablas, gráficos, etc., exactamente lo mismo que hacen ciertos software de investigación, como el Atlas-ti. Por tanto, close reading y distant reading son en realidad formas complementarias.

De todo este arsenal de datos se exige igualmente que el crítico elucide patrones e informaciones significativas, es decir, hace falta un trabajo de síntesis. En cierto modo, lo que hay tras la distant reading es lo mismo que en la close reading, aunque con estrategias bien distintas: se trata de un modelo conversacional, dialógico, de enfrentarse a la lectura para extraer su potencial de sentido, aunque la distant reading lo hace con las "gafas de lejos" y la close reading con las de cerca, si se nos permite la analogía. En clave cinematográfica, aquélla exige un encuadre muy amplio, con un travelling sostenido; en cambio, la close reading se parece más a un primer plano, a una forma más bien teatral o fotográfica, en el sentido de que se concentra en un escenario predelimitado.

Sea como sea, el problema de la lectura atenta es que se centra en un canon reducido, lo cual contrasta con la pluralidad de la literatura actual y la necesidad del lector hipertextual de "surfear" muchos textos. Ciertamente, la consideración de los textos canónicos privilegiados como algo sacralizado, como objetos de "unicidad", según la estilística, oculta lo que comparten con otros objetos literarios: mecanismos, temas, tropos, géneros o sistemas. Por ejemplo, el Quijote comparte rasgos de la escritura carnavalesca diseminados por toda la obra: chanzas, disfraces, irreverencias, juegos de sentido, etc. Podemos optar por diversos enfoques de aproximación, pero si, por ejemplo, analizamos los capítulos que tratan de la cueva de Montesinos, hemos de saber que estos rasgos no sólo se hallan ahí, sino que están diseminados por muchos otros fragmentos y textos tanto cervantinos como de la época.

La perspectiva es diametralmente distinta: el canon clásico tiende a crear una taxonomía jerarquizante donde el foco de interés eran los grandes clásicos o genios del pensamiento y la literatura y, en un segundo nivel, de sus discípulos o continuadores, una vez delimitados los contornos de una escuela o tendencia estética. La historiografía literaria reposa siempre en una axiología que conforma una ordenación jerárquica, basada a su vez en una forma de construcción o especulación metaliteraria. Porque, más adelante, sabemos que la crítica cambia sus juicios o que la recepción del público ensalza o rebaja la posición relativa que se había dado a una lectura entre sus contemporáneos. Digamos, pues, que con el tiempo se recupera una visión más pragmática, es decir, parafraseando a Eco, se invocan los textos no sólo como signos interpretados con mayor o menor eco o acierto, sino que se trata de trascenderlos para analizar lo que el documento nos revela de su época. 
En una línea similar a Moretti, Lev Manovich (2002) trata de analizar los artefactos digitales de los nuevos media para visualizar patrones significativos, es lo que se ha llamado cultural analytics.

\section{La aportación de Mignolo}

La lectura distante busca modelos explicativos que describan la literatura como un sistema, la lectura cercana busca la interpretación desde un texto concreto. El semiólogo Mignolo (1983) señala dos perspectivas distintas del estudio literario: la comprensión bermenéutica y la comprensión teórica.

La analogía es clara: cuando por ejemplo abordamos una lectura cualquiera tenemos en primer lugar que comprenderla, esto es, interpretarla conforme una serie de "normas" discursivas, de género, etc. que configuran el "horizonte de expectativas" del lector; otro segundo nivel de lectura es el de la comprensión teórica, que trata de explicar el texto y para ello indaga en qué grado se verifican en él determinados modelos o teorías. No es lo mismo, por ejemplo, tratar de entender las convenciones de lectura o de una película de una ficción de Tolkien, que analizarlas en función de modelos subyacentes.

Señala Mignolo (1983) que el formalismo y la gramática generativa dieron los primeros pasos al reemplazar la noción de la obra por la de sistema. Así, desde el punto de vista de la investigación textual, "el objeto de la teoría se postula como una construcción que consiste en seleccionar y explicar las propiedades relevantes de todo texto y de los textos literarios en particular" (19).

En consecuencia, la comprensión hermenéutica se orienta a la interpretación de obras particulares y se relaciona con una "comunidad interpretativa" de lectores, a diferencia de la comprensión teórica, orientada hacia los problemas de explicación y relacionada con una "comunidad científica". La praxis cultural de estas últimas décadas ha dado la razón a Mignolo (1983): los lectores como masa de consumidores han seguido dinámicas que forman parte de periferias culturales (por ejemplo, el fanfiction, los superhéroes, etc.) y la Academia ha seguido otras vías en sus pretensiones de describir la cultura y la literatura (no una obra $\mathrm{x}$ ) con métodos científicos.

La lectura distante participaría de este enfoque, mientras que la lectura cercana es una lectura situada, esto es, que participa de los parámetros de una comunidad interpretativa dada, igual que un rabino cuando propone un comentario o midrash de un texto bíblico comparte una serie de códigos de interpretación. Porque interpretar, en este sentido, es comprender de forma participativa, y por eso mismo cabe la empatía, la aceptación o el rechazo, cosa que difícilmente se produce cuando lo que elucidamos es un volumen de datos sobre una serie de textos. 
Apoyándose en A. Schutz, Mignolo (1983) destaca una comprensión de primer grado y una comprensión de segundo grado, que se corresponde con lo descrito anteriormente: la primera se realiza bajo la forma de participación y la segunda de observación. Dicho de modo más sencillo, en la primera actuamos como lectores/oyentes que nos acercamos a Hansel y Gretel o La Atlánti$d a$ de Benoit. La participación se evidencia no ya en las normas intersubjetivas de comprensión que nos hacen adscribir estos relatos a unas clases convenidas ("cuento", "novela",...), sino en la propia dimensión patética de dichas historias: "la muerte -en palabras de Benjamin (1986: 202) - es la sanción de todo lo que puede relatar el narrador". Dicho de otro modo, las lecturas tienen una dimensión convivencial, emocional, que fomenta nuestra complicidad.

En cambio, en la comprensión teórica actuamos como lo hizo Propp ([1928] 1974) al estudiar los cuentos maravillosos rusos, intentando explicar una serie de regularidades apreciables en todos esos textos en función de unos instrumentos de análisis preestablecidos. Verificar que una estructura se da en un texto (ya sea una estructura retórica, narratológica, ideológica, etc.) es hacerlo predecible, convertirlo en espejo de ese patrón que queremos evidenciar. Por ejemplo, leer los cuentos de hadas según el modelo narratológico de Propp ([1928] 1974) supone describir unas determinadas secuencias o funciones, y si pasamos al plano antropológico, supone identificar en los cuentos los pasos el rito de iniciación. En un caso la actividad principal es la de participar, en la segunda la de observar, organizando el conocimiento en conceptos que se trasladan para el análisis.

La paradoja de una lectura distante es que una aproximación a los textos sin ese carácter experiencial y participativo nos impide lo que es esencial en el lector experto, que es (re) significar la obra a partir de una construcción activa del sentido y nos vuelve a los métodos de un lector ingenuo, que procesa todo de la misma manera. Por ejemplo, si atendemos a etiquetas o metadatos, el Quijote es una novela de caballerías, y por tanto entraría en la seriación de obras de este género, pero si entendemos que "no" es una novela al uso sino una parodia bajtiniana del género (Bajtin, 1974), entonces su seriación es precisamente con otros textos de escritura carnavalesca.

Los libros, podríamos decir, son artefactos con un diseño inteligente y por tanto se prestan a estos diversos enfoques. Participar de un texto es apropiarse del mismo, y en esa línea están teóricos como Chartier (1992), pero también la propia praxis social de la customización o personalización de lecturas. Por el contrario, el rol de observador exige un entrenamiento y una despersonalización poco común en, por ejemplo, el lector hipertextual.

Si lo que buscamos son leyes o principios explicativos (enfoque nomotético) cabe decir que esto interesa a la comunidad científica pero no a la 
comunidad de lectores. La Academia persigue descubrimientos o corroborar hipótesis científicas que sólo lo son en la medida en que son confirmados y sancionados por otros miembros de la comunidad científica. La comprensión teórica requiere pues una observación distanciadora del fenómeno, pero la comprensión hermenéutica implica necesariamente participar en él.

Por otra parte, la necesidad de una alfabetización situada, según recomienda la Unesco, pone el énfasis en los textos cercanos, en el sentido de próximos al entorno del alumno. Lo próximo no es el género, por ejemplo, que no deja de ser una forma de acuñación conceptual, sino los elementos de una memoria cultural compartida, ya sea un texto, una leyenda, un autor, un evento, etc.

Toda época ha dispuesto de mecanismos metatextuales para categorizar sus propios textos, pero eso surgía de la experiencia de sus textos, no del programa de una computadora. De hecho, estamos viviendo nuevos fenómenos en este sentido: el turismo recupera tradiciones o leyendas para impulsar un destino, y todo ello vuelve a poner en marcha el "flujo" de textos, que Menéndez Pidal (1963) llamaba sabiamente el "estado latente" de la tradición. No podemos descartar nuevas lecturas de los textos o géneros conocidos que emerjan o se pongan de moda por determinados factores. Así, la subcultura gótica ha "visibilizado" muchos textos y manifestaciones que antes sencillamente hubieran sido tildados de grotescos.

\section{Sintesis}

En realidad, el modelo que preconiza la distant reading es utilizado por motores de búsqueda y robots que procesan gran cantidad de libros para recomendar nuevas lecturas. Es lo que hace Amazon cuando tras cualquier compra filtra las afinidades de cada lector y le recomienda otros libros "parecidos" que han comprado otros consumidores. Es, en suma, una orientación al consumidor pero también se presenta a veces como un apoyo heurístico para sugerir próximas lecturas.

Es lo que hacen numerosos websites como GoodReads, Shelfari, Whichbook, Library Thing, Yournexread, etc. Como botón de muestra, tenemos el caso de Gnooks, un robot que encuentra lineamentos de otros autores más o menos conexos a nuestra elección. En este caso, las sugerencias hacia Phil K. Dick enriquecen sin duda al lector que hace la búsqueda, si bien en otros casos (si probamos con Juan Rulfo o Federico García Lorca) las conexiones que se hallan son bastante cuestionables. 


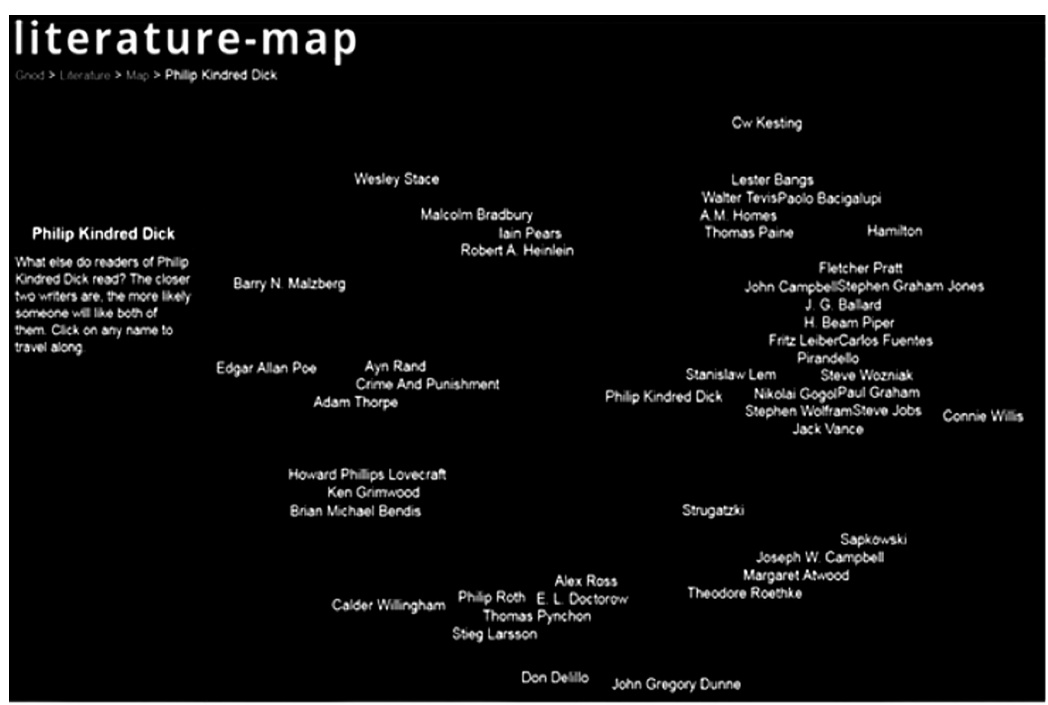

Figura 1. Gráfico de la escritora J. K. Rowling (Gnod)

Fuente: http://catolink4.blogspot.com.es/2012/01/literature-map.html

En todo caso, pueden ser instrumentos útiles en campos como la literatura infantil y juvenil donde los registros son innumerables y se hace necesario "refinar" los criterios de búsqueda. De todos modos, no es solamente un problema de estrategia informacional: en un nivel previo tiene que estar un mediador de lectura, esto es, un profesor o experto que sepa orientar en las categorizaciones. Por citar un caso emblemático, en cualquier búsqueda acerca de Tolkien, Lewis u otros autores de sagas, encontraremos que el mismo concepto de saga no está bien catalogado, pues una saga no se formaría simplemente de las narrativas épicas tradicionales, o bien, modernamente, de la literatura fantástica, sino que sería ante todo una narración serial (Martos García, 2009a).

\section{Prospectiva}

\section{Un modelo proactivo: la comunidad de lectores como comunidad de fans}

Siguiendo la exposición de Martos García (2009b), cabe decir que los jóvenes del siglo XXI están inmersos en una cultura mediática y digital. Por eso 
mismo su relación con la cultura letrada es por canales distintos: la cultura académica/escolar, que es asimilada de forma limitada, como una exigencia profesional, y la cibercultura o los medios, por ejemplo, la industria del entretenimiento, que va a manejar clásicos o ficciones propias de la literatura de aventuras, ciencia ficción, fantasía, etc. La recepción en estos casos es entonces activa, porque van a formar parte de las preferencias y de los gustos compartidos por grupos de jóvenes, que, como los que se podrían adscribir dentro de los geek, fandom, góticos..., convierten tales textos en origen de la cultura letrada, como son muchas mitologías modernas, y en parte o nutriente de su propia subcultura y en modas de amplio alcance. Todo eso se ha expandido gracias a Internet, a la cultura digital, y no tanto a la cultura audiovisual clásica (películas, radio, periódicos) que apenas dejaba cauces a la participación, a la colaboración o a la creación activa de redes de lectores, que reivindican sus propias aportaciones.

El fanfiction, en conexión con las sagas y la ficción fantástica en general, es un fenómeno que ha sido estudiado ampliamente por Martos García (2009b). Se puede decir que es un movimiento de recreación de los textos de universos de ficción que cobran fama entre los jóvenes y son objeto, pues, de veneración, esto es, se convierten en universos compartidos. Son textos a-propiados, revisitados por los jóvenes, que actualizan sus tramas y discursos conforme a sus propias preferencias.

Los mecanismos de imitación son muy libres, sólo hay que respetar, por un convenio tácito, un mismo punto de partida, lo que se conoce como la versión canónica de la saga o historia de origen, luego todo lo demás es susceptible de ser variado y completado con precuelas, secuelas, variaciones de todo tipo. Es el mismo mecanismo de los faces y pastiches en las fotos y recreaciones de cuadros.

Según Martín Barbero (1999: 89-90), se ha formado un nuevo sensorium a partir de la hegemonía de la televisión, de las modas audiovisuales y de las manifestaciones de lo que se conoce como posmodernidad, que es una elaboración sincrética y sinestésica de las formas sensoriales heterogéneas que provenían lo mismo del arte que de la publicidad o la industria, tal como vemos en la cultura pop.

Este nuevo sensorium audiovisual (Idarraga Franco, 2009) está marcado por las experiencias de nomadismo, instantaneidad y flujo, es decir, por una presencia que se ramifica en manifestaciones diversas y efímeras todas. No olvidemos que estamos ante la cultura de la fragmentación, ante la cultura mosaico (Moles, 1971), ante la segmentación de audiencia que impone la cultura digital, que hace que se multipliquen los escenarios y los performances en un sentido en espiral. Lo cierto es que los fans practican un tipo de lectura 
entusiasta, casi compulsiva, que se relaciona en parte con las corrientes críticas y en parte con los mismos mecanismos de banalización y mercantilización de la cultura.

La cultura fan, por tanto, no puede desligarse del consumo, pero a la vez es una lectura alternativa a la lectura orientada desde o por la Academia. Como lectura empática, es siempre una lectura participativa, compartida, socializada y que no busca el distanciamiento de su corpus textual, al contario.

De un modo u otro, la forma de recuperar la lectura cercana en toda su virtualidad no es la de contraponer lecturas extensivas e intensivas, tampoco es volver a propuestas de estudio monográfico sobre un autor o una obra, como en los programas clásicos. Estas comunidades de fans (fandom) nos enseña al respecto algunas pautas interesantes: los espacios de lectura no se sustentan solamente en artefactos como los libros, en lugares como las bibliotecas, o en programas como los que subyace a la lectura conectada: lo que importan son las interacciones y la socialización de la lectura, es decir, un modelo conversacional, dialógico. De ahí que se popularicen prácticas como los cosplay, los fanzines, las historias de fanfiction, las convenciones temáticas, los fanart, el fansub, o la colección de merchandising relativa al objeto de su admiración.

\section{Hacia un lector expandido y experto}

El modelo de educación expandida, sensible a la transdisciplinariedad, a la participación ciudadana y la conciencia planetaria (Morin y Pakman, 1994), desde un enfoque lúdico y ambital, trata de definir proyectos de intervención tecnosocial, cercanos a un activismo social que se relaciona con lo contracultural en educación (edupunk), pero que en todo caso se presenta con una actitud creativa y experimental muy reconocible en prácticas instituidas ya, como las comunidades de aprendizaje (Figura 2).

De hecho, en las sagas fantásticas se habla de universo expandido para referirse a los otros ámbitos a los que "migra" una ficción, por ejemplo, la saga de Tolkien ha pasado a videojuegos, cartas, disfraces, juguetes y un sinfín de prácticas sociales, muchas de ellas alternativas. La lectura, ya sea cercana o alejada, tiene que ser extrapolable a otros ámbitos y retraducida a otros lenguajes.

Por tanto, en el ámbito de la lectura, el desideratum es pasar igualmente de un lector ingenuo y sumiso a un lector disidente y experto, esto es, rico en experiencias de lectura, formado, y por tanto con criterios suficientes para elegir y decidir itinerarios de lectura más allá de las campañas de marketing o las actitudes "meméticas" que subyacen a los videos virales. Por otro lado, el lector experto es siempre al final un "escrilector”, es decir, un prosumidor. 


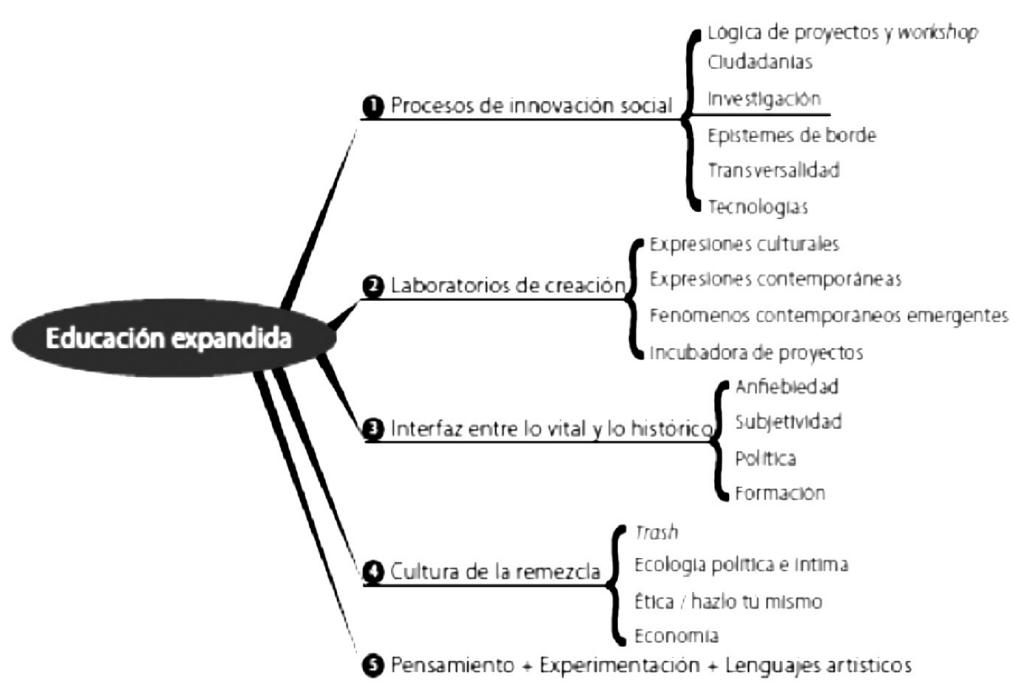

Figura 2. Gráfico de A. F. Díaz sobre educación expandida

Fuente: Díaz (2011: 79)

Sin duda el ejercicio de la lectura y la escritura van juntos, por tanto debemos fomentar prácticas de escritura amateur y creativa, como el fanfiction, si bien separándola de aquellas prácticas cercanas al circuito comercial, como el fanadvertising. En realidad, en el mundo de las lecturas de éxito, cada vez es más difícil separar lo publicitario o promocional, la comunicación espontánea entre lectores y fans, y el comentario o la reseña, de ahí la necesidad de potenciar un tipo de lector experto.

\section{Conclusiones}

De todo lo dicho, podemos concluir que cada vez es más complicado establecer los límites entre unas modalidades de lectura y otras, y que las nuevas prácticas culturales van en el camino de promover una lectura más intermedial y sinestésica (concepto de sensorium).

El textocentrismo de las escuelas clásicas, que reducía la lectura a la explicación de textos impresos, se ve ahora por aproximaciones como la de Moretti o Manovich, que tratan de visualizar patrones culturales, es decir, trascender el texto para analizar el contexto. Siguiendo el análisis propuesto por Manovich (2011), proyectos de bases de datos como Tropes.tv (2012) evidencian 
que se ha invertido el principio paradigma-sintagma: ahora la base de datos es lo que tiene existencia material, y el sintagma, las narrativas potenciales, es lo que se presenta desmaterializado.

La síntesis necesaria es, pues, la integración de todos estos textos-mosaico, la "desfragmentación", reuniendo estas piezas diversas en conjuntos significativos. Lo que importa no son los puntos sino las conexiones entre esos puntos, o, dicho de otro modo, qué somos capaces de extraer de esos metadatos desde perspectivas reintegradoras, por ejemplo, la etnografía de la comunicación, la teoría de juegos o, en general, la vastedad de campos que están empezando a abordar las Humanidades Digitales (Galina-Russell, 2011). Todo ello llevará a un renacimiento de una lectura que dialécticamente supere la close reading y la distant reading, o también lo que se conoce como lectura de "surfeo" propia del hipertexto.

Los polos tesis-antítesis deben generar una síntesis conciliadora, una lectura disidente, crítica, inclusiva y heurística, capaz de cultivar la imaginación y la creatividad como formas de acceder a una ciudadanía que ya no es la de unos pocos estudiosos que escrutan un texto clásico en forma de lectura intensiva, sino la de una inteligencia colectiva, que es cada vez más híbrida y global en sus manifestaciones étnicas, culturales o económicas. Por ello, resignificar los textos, el patrimonio cultural o la cultura urbana forma parte de un mismo impulso en que la lectura será un eje determinante, a pesar de los intentos de banalización o mercantilización extrema.

\section{REFERENCIAS}

Bajtin, M. 1974. La cultura popular en la Edad Media y Renacimiento. España: Barral. Benjamin, W. 1986. "El narrador. Consideraciones sobre la obra de Nicolai Leskov". Sobre el programa de la filosofía futura, 189-212. Barcelona: Planeta Agostini.

Besson, A. 2004. D'Asimov à Tolkien: cycles et séries dans la littérature de genre. Francia: CNRS.

Bolter, J. D., R. Grusin y R. A. Grusin. 2000. Remediation: Understanding new media. EUA: MIT Press.

Chartier, R. 1992. El mundo como representación: estudios sobre historia cultural. Barcelona: Gedisa.

Díaz, A. D. F. 2011. "Educación expandida y cultura digital. Una exploración de proyectos tecnosociales en Colombia”. Hallazgos 8: 71-90. revistas.usta.edu.co/index.php/hallazgos/article/download/1696/1845

Dufays, J. L., L. Gemenne y D. Ledur. 2005. Pour une lecture littéraire: histoire, théories, pistes pour la classe. París: De Boeck Supérieur.

Galina-Russell, I. 2011. “¿Qué son las humanidades digitales?”. Revista digital universitaria $12(7): 3-10$. 
Idarraga Franco, H. F. 2009. "Sensorium e internet; una aproximación al fenómeno tecnológico desde la obra de Walter Benjamin", tesis doctoral, Colombia: Pontificia Universidad Javeriana.

Jenkins, H. 2006. Convergence culture: la cultura de la convergencia de los medios de comunicación. Barcelona: Paidós

Lovink, G. 2013. Zero comments: Blogging and critical Internet culture. UK: Routledge.

Manovich, L. 2002. "New media from Borges to HTML". The new media reader 1: 13-28.

Manovich, L. 2011. "Trending: The promises and the challenges of big social data". Debates in the digital bumanities 2: 460-475.

Martín-Barbero, J. 1999. "Recepción de medios y consumo cultural: travesías”, en El Consumo Cultural en América Latina, Guillermo Sunkel, coord., 47-71. Colombia: Convenio Andrés Bello.

Martos García, A. E. 2009a. Introducción al mundo de las sagas. Badajoz: Universidad de Extremadura.

Martos García, A. E. 2009b. "Tecnologías de la palabra en la era digital: de la cultura letrada a la cibercultura”. RELATEC: Revista Latinoamericana de Tecnología Educativa 8 (2): 15-37.

Martos García, A. y A. E. Martos García. 2014. "Las nuevas escrituras juveniles en el contexto de la posmodernidad". Textura-ULBRA 15 (29): 3-17.

Martos Núñez, E. y A. E. Martos García. 2014. "Textos integrados y narratologías míticas y posmodernas: algunos paralelismos". Revista chilena de literatura 87: 183-211.

Menéndez Pidal, R. 1963. "El estado latente en la vida tradicional". Revista de Occidente 2: 129-151.

Mignolo, W. D. 1983. "Comprensión hermenéutica y comprensión teórica”. Revista de Literatura Madrid 45 (90): 5-38.

Moles A. 1971. Sociodinamica della cultura. Bologna: Guaraldi.

Moretti, F. 2000. "Conjectures on World Literature”. New Left Review 1: pp. 54-68.

Moretti, F. 2013. Distant Reading. Londres: Verso.

Moretti, F. 2005. Graphs, Maps, Trees: Abstract Models for Literary History. Londres: Verso.

Morin, E. y M. Pakman. 1994. Introducción al pensamiento complejo. Barcelona: Gedisa.

Morin, E., E. R. Ciurana y R. D. Motta. 2002. Educar en la era planetaria: el pensamiento complejo como método de aprendizaje en el error y la incertidumbre bumana. Valladolid: Universidad de Valladolid, Secretariado de Publicaciones e Intercambio Cultural.

Propp, V. (1928) 1974. Las raíces históricas del cuento. Madrid: Editorial Fundamentos.

TVTropes. 2012. TV Tropes Foundation. http://tvtropes.org/

\section{Para citar este texto:}

Martos Núñez, Eloy y Aitana Martos García. 2018. "Categorizaciones de la lectura y praxis cultural en la era digital: distant reading vs. close reading”. Investigación Bibliotecológica: archivonomía, bibliotecología e información 32 (74): 19-33. http://dx.doi.org/10.22201/iibi.24488321xe.2018.74.57904 